\title{
MALESTAR EMOCIONAL ASOCIADO A LA ORIENTACIÓN SEXUAL EN PERSONAS MAYORES
}

\author{
Juan Enrique Nebot-García \\ Salusex-Unisexsida. Departamento de Psicología Básica, Clínica y Psicobiología, Universitat Jaume I de \\ Castellón. \\ junebot@uji.es \\ Cristina Giménez-García \\ Salusex-Unisexsida. Departamento de Psicología Básica, Clínica y Psicobiología, Universitat Jaume I de \\ Castellón. \\ Estefanía Ruíz-Palomino \\ Salusex-Unisexsida. Departamento de Psicología Básica, Clínica y Psicobiología, Universitat Jaume I de \\ Castellón. \\ Paula Iglesias Campos \\ Salusex-Unisexsida. Departamento de Psicología Evolutiva y de la Educación, Universitat de València. \\ Rafael Ballester-Arnal \\ Salusex-Unisexsida. Departamento de Psicología Básica, Clínica y Psicobiología, Universitat Jaume I de \\ Castellón.
}

\section{RESUMEN}

Introducción. En la actualidad, pese a haber avanzado en los derechos legales de las personas con diversidad afectivo-sexual, siguen habiendo prejuicios sociales al respecto. Este hecho se ve multiplicado en las personas mayores, que han sufrido una educación más homófoba y una mayor invisibilización de su sexualidad, por lo que se hace necesario investigar también en esta población.

Método. Participaron 139 personas, de entre 50 y 73 años, con una media de 56,1 años (DT=5,44), siendo el $40,3 \%$ mujeres y el $59,7 \%$ hombres. El $89,9 \%$ se definían como heterosexuales y el $10,1 \%$ como no heterosexuales. Todos cumplimentaron un cuestionario sobre la vivencia de su orientación sexual.

Resultados. El $50 \%$ de personas no heterosexuales, como consecuencia de su orientación sexual, se ha sentido rechazado, el $42,9 \%$ ha experimentado malestar, el $28,6 \%$ sufrió violencia verbal y el 14,3\% violencia física. Así, se observan diferencias significativas entre las personas no heterosexuales y las heterosexuales en diversos aspectos relacionados con su orientación sexual, siendo las no heterosexuales las que muestran mayor malestar emocional $\left(\chi^{2}=20,61 ; p<, 000\right)$, más dudas con su orientación ( $\left.{ }^{2}=15,01 ; p<, 000\right)$, han sufrido más violencia verbal $\left(\chi^{2}=18,03 ; p<, 000\right)$ y más violencia física $\left(\chi^{2}=18,11 ; p<, 000\right)$.

Conclusiones. Estos hallazgos confirman que el rechazo social de algunas orientaciones sexua- 


\section{MALESTAR EMOCIONAL ASOCIADO A LA ORIENTACIÓN SEXUAL EN PERSONAS MAYORES}

les puede suponer una fuente de malestar emocional, así como un factor de riesgo para ser víctima de la violencia. En general, las personas con una orientación no heterosexual mostrarían mayor afectación emocional y mayor probabilidad de sufrir agresiones. Con el fin de mejorar esta situación, cabría analizar qué variables pueden mediar en estos resultados para establecer estrategias adecuadas de prevención e intervención a lo largo de todo el ciclo vital.

Palabras clave: Personas mayores; orientación sexual; malestar emocional; violencia

\section{ABSTRACT}

Emotional discomfort associated with sexual orientation in the elderly. Introduction. At present, despite some advances in the legal rights of people with sexual-affective diversity, there are still related social prejudices. This fact is multiplied in the elderly, who have suffered a more homophobic education and a greater invisibility of their sexuality, so it is necessary to investigate in this population too.

Method. 139 people participated, between 50 and 73 years old, with an average of 56.1 years old ( $S D=5.44)$, being $40.3 \%$ women and $59.7 \%$ men. $89.9 \%$ self-defined as heterosexual and $10.1 \%$ as non-heterosexual. All of them completed a questionnaire about the experience of their sexual orientation.

Results. $50 \%$ of non-heterosexual people have felt rejectiondue to their sexual orientation, $42.9 \%$ have experienced discomfort, $28.6 \%$ suffered verbal violence and $14.3 \%$ physical violence. Thus, significant differences are observed between non-heterosexual and heterosexual people in various aspects related to their sexual orientation, being non-heterosexual those who show greater emotional distress $\left(\chi^{2}=20.61, p<.000\right)$, more doubts about their orientation $\left(\chi^{2}=15.01 ; p<.000\right)$, suffering more verbal violence $\left({ }^{2}=18.03 ; p<.000\right)$ and more physical violence $\left(\chi^{2}=18.11 ; p<.000\right)$.

Conclusions. These findings confirm that the social rejection of some sexual orientations can be a source of emotional discomfort, as well as a risk factor to be a victim of violence. In general, people with a non-heterosexual orientation would show greater emotional affectation and greater probability of suffering aggressions. In order to improve this situation, we should analyze what variables can mediate in these results to establish adequate prevention and intervention strategies throughout the life cycle.

Keywords: elderly people; sexual orientation; emotional discomfort; violence

\section{INTRODUCCIÓN}

La sexualidad es una dimensión importante para la calidad de vida de las personas (Diamond \& Huebner, 2012; Lindau \& Gavrilova, 2010). Las falsas creencias y los mitos sobre la sexualidad (Wynn, Foster \& Trussell, 2009), un tema que todavía es considerado tabú (Morgan, Thorne \& Zurbriggen, 2010), han condicionado la vivencia de la sexualidad de la población en general, y más aún en la gente mayor (Herek \& McLemore, 2013), cuya sexualidad ha sido invisibilizada tradicionalmente (Lindau et al., 2007).

La diversidad sexual ha sido uno de los aspectos más invisibilizados, como consecuencia de la heteronormatividad imperante (Herz \& Johansson, 2015), es decir, la presunción de que todas las personas son heterosexuales, hasta que no se demuestre lo contrario. Esta creencia se expresa de diferentes modos, como la forma en que se abordan los contenidos en la educación formal (DePalma \& Jennett, 2010; McNeill, 2013; Röndahl, 2011), el trato que se recibe en la sanidad (Enson, 2015; Hayman, Wilkes, Halcomb \& Jackson, 2013; Morrison \& Dinkel, 2012), las problemáticas que se investigan desde la ciencia (Herz \& Johansson, 2015) e, incluso, en las leyes que se adoptan (Asociación Internacional de Lesbianas, Gays, Bisexuales, Trans e Intersex, 2017).

Esta influencia normativa también se ve reflejada en el acoso sufrido por las personas LGTBI 
(lesbianas, gays, transexuales, bisexuales e intersexuales). De hecho, un $49,2 \%$ ha sido víctima de insultos y un $13,1 \%$ ha sufrido violencia física debido a su orientación sexual (Herek, 2009). Además, un 38\% de población LGTBI, alguna vez en su vida, se ha sentido discriminada por su orientación sexual o su identidad de género (Digoix et al., 2016). En cuanto a la población mayor no heterosexual, un $63 \%$ ha sufrido violencia verbal y un $29 \%$, violencia física (D’Augelli \& Grossman, 2001). En consecuencia, algunos estudios han encontrado en el colectivo LGTBI, mayores niveles de depresión (Carroll, 2015; Espada, Morales, Orgilés \& Ballester, 2012; Marchueta, 2014; Martxueta \& Etxeberria, 2014) y de ansiedad (Marchueta, 2014; Martxueta \& Etxeberria, 2014), y mayor ideación suicida (Carroll, 2015; Reisner, Biello, Perry, Gamarel \& Mimiaga, 2014).

Si nos centramos en población mayor, tal y como ocurre con su sexualidad en general, la orientación sexual es un tema escasamente estudiado (Fredriksen-Goldsen \& Kim, 2015). En los pocos estudios que existen, se observa que la población mayor LGTB, en comparación con sus pares heterosexuales, presenta unos niveles de depresión y malestar psicológico más elevados, peor funcionamiento cognitivo y más dificultad con las actividades instrumentales de la vida diaria (FredriksenGoldsen, Kim, Barkan, Muraco \& Hoy-Ellis, 2013; Gonzales \& Henning-Smith, 2015; Seelman, 2018), lo que parece lógico dado que a las causas de la discriminación que sufre la población joven LGTBI hay que añadir el que las personas mayores han sido socializadas en un entorno mucho más homófobo que los jóvenes.

Ante este panorama, resulta imprescindible analizar la experiencia de la orientación sexual de la población en general y, de la más adulta, en particular. Es por ello que, en este estudio, se pretende dar visibilidad a la vivencia que tienen las personas mayores respecto a su orientación sexual y a la discriminación recibida.

\section{OBJETIVO}

Analizar la vivencia de la propia orientación sexual de personas adultas mayores y de la violencia recibida como consecuencia de su orientación sexual.

\section{PARTICIPANTES}

En el presente estudio participaron un total de 139 personas, de entre 50 y 73 años, con una media de 56,1 años (DT=5,44), siendo el $40,3 \%$ mujeres y el $59,7 \%$ hombres. El $89,9 \%$ se definían como heterosexuales y el 10,1\% como no heterosexuales. Los criterios de inclusión eran: tener más de 50 años y residir en España.

\section{INSTRUMENTOS DE EVALUACIÓN}

Para este estudio, además de las preguntas sobre los datos sociodemográficos, se utilizó un cuestionario ad hoc realizado por nuestro grupo Salusex-Unisexsida, que explora la diversidad afectivo-sexual y la discriminación debido a orientación sexual. Para este trabajo se utilizaron 4 ítems, referidos a los siguientes aspectos:

Dudas con la propia Orientación Sexual. En esta escala se pregunta si «A lo largo de los años, ¿has dudado sobre tu orientación sexual?». Los participantes debían responder siguiendo una escala tipo Likert de 0 (Nada) a 3 (Mucho). Para facilitar el análisis de los resultados, se agruparon aquellas respuestas que, en mayor o menor grado, habían mostrado algún tipo de duda con la propia orientación sexual (1=Algo, 2=Bastante 0 3=Mucho).

Malestar asociado con la Orientación Sexual. Este aspecto se explora con dos ítems. En el primero se pregunta «A lo largo de los años, ¿cuánto malestar has sentido sobre tu orientación sexual?». Los participantes han de contestar siguiendo una escala tipo Likert de 0 (Nada) a 3 (Mucho). Aquellas respuestas que muestran algún grado de malestar (1=Algo, 2=Bastante 0 


\section{MALESTAR EMOCIONAL ASOCIADO A LA ORIENTACIÓN SEXUAL EN PERSONAS MAYORES}

3=Mucho), se han reagrupado para analizar más fácilmente los resultados. En segunda instancia, a las personas que han mostrado algún grado de malestar, se les pregunta también qué tipo de malestar han experimentado, pudiendo contestar entre «sentirse rechazado/a», «sentirse solo/a», "sentirse como un/a enfermo/a»; "sentirse como un/a vicioso/a» 0 «sentirse culpable».

Violencia recibida debido a su Orientación Sexual. Para explorar esta cuestión se utilizaron dos ítems, uno que evalúa violencia verbal recibida: «¿Has sufrido alguna vez una agresión verbal debido a tu orientación sexual?»; y otro que mide violencia física recibida: «¿Has sufrido alguna vez una agresión física debido a tu orientación sexual?». En ambos ítems se debe responder con un «Sí»» 0 un «No».

\section{PROCEDIMIENTO}

Este estudio forma parte de un proyecto de investigación más amplio que analiza la orientación sexual, de manera comprehensiva, en distintas etapas del ciclo vital. Como primer paso, se confeccionó un cuestionario ad hoc sobre la vivencia de la orientación sexual, creando también una versión on line. Los participantes conocieron el estudio mediante redes sociales y foros online de interés. Una vez estaban interesados, accedían al formulario online para dar su consentimiento y, seguidamente, poder cumplimentar el cuestionario. La participación era anónima, voluntaria y confidencial.

\section{ANÁLISIS ESTADÍSTICOS}

En primer lugar, con el programa estadístico SPSS versión 24, se realizaron análisis descriptivos de las principales variables estudiadas. Asimismo, para analizar las diferencias según la orientación sexual, se realizaron pruebas de Chi cuadrado.

\section{RESULTADOS}

Como habíamos dicho en el apartado referido a los "Participantes", el 89,9\% se definieron como heterosexuales y el 10,1\% como no heterosexuales. La población mayor no heterosexual, en comparación con sus pares heterosexuales, muestra prevalencias mayores al experimentar dudas y malestar relacionado con su orientación sexual (ver figura 1). En ambos casos las diferencias fueron significativas, tanto en las dudas $\left(\chi^{2}=15,01 ; p<, 001\right)$ como con el malestar asociado $\left(\chi^{2}=20,61\right.$; $p<, 001)$. No obstante, hay que remarcar ese $8 \%$ de personas que se definen como heterosexuales pero que han experimentado dudas al respecto y también el $42,9 \%$ de personas no heterosexuales que se han sentido mal como consecuencia de su orientación sexual.

Figura 1. Porcentaje de personas que han experimentado dudas o malestar con su orientación sexual.

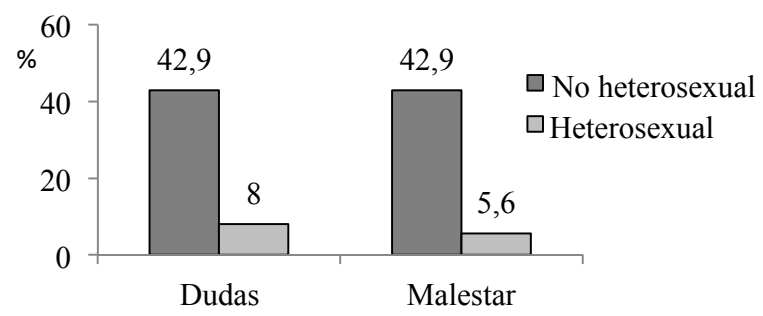

Entre las personas participantes que informan haber sentido malestar debido a su orientación sexual, las personas no heterosexuales informan con mayor frecuencia de los distintos tipos de 
malestar ocasionado (ver figura 2), a excepción de las variables «vicioso/a» $\left(\chi^{2}=0,929 ; p<, 335\right)$ y «enfermo/a». Sin embargo, solamente se alcanzan diferencias significativamente estadísticas en sentirse «rechazado/a» $\left({ }^{2}=4,55 ; p<, 033\right)$, pero no en sentirse «solo/a» $\left(\chi^{2}=0,06 ; p<, 797\right) 0$ «culpable» $\left(\chi^{2}=0,03 ; p<, 853\right)$.

Figura 2. Porcentaje de personas que han experimentado cada tipo de malestar.

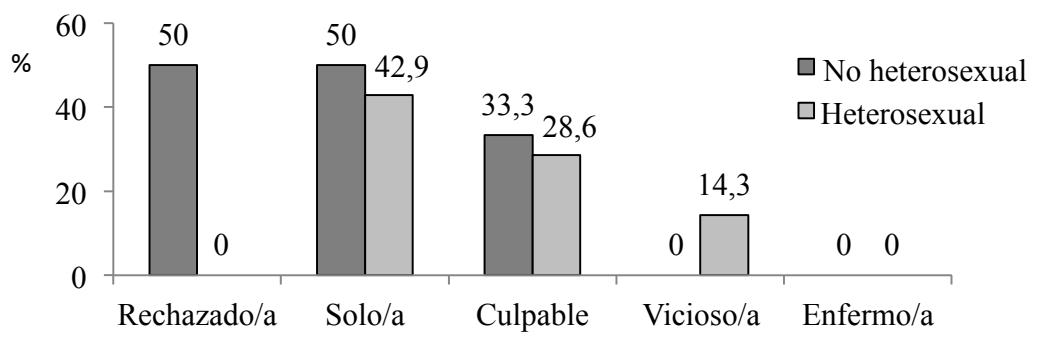

Por lo que respecta a la violencia sufrida debido a su orientación sexual, la población no heterosexual presenta mayores niveles en ambos tipos de violencia (ver figura 3), observándose diferencias significativas tanto en la violencia verbal $\left(\chi^{2}=18,03 ; p<, 001\right)$ como en la física $\left(\chi^{2}=18,11\right.$; $\mathrm{p}<, 001)$.

Figura 3. Porcentaje de personas que han sufrido violencia verbal y física debido a su orientación sexual.

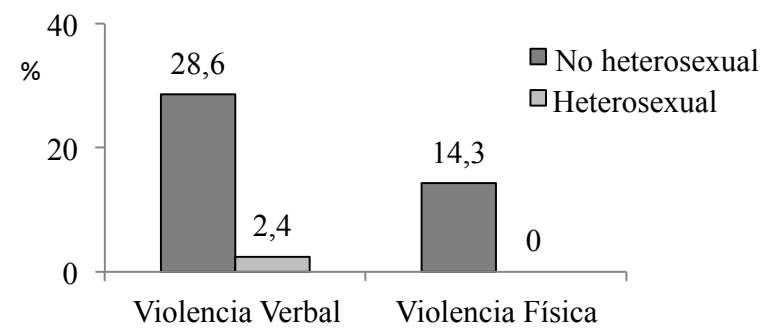

\section{CONCLUSIONES}

En primer lugar, a partir de nuestros hallazgos, se visibilizaría la existencia de una mayor diversidad afectivo-sexual entre las personas mayores, al igual que en pasados estudios (Herbenick et al., 2010), que restaría credibilidad a la visión heteronormativa imperante (Herz \& Johansson, 2015). En segundo lugar, se observa en qué medida la población mayor no heterosexual muestra mayores niveles de malestar y dudas con su orientación sexual, obteniéndose diferencias significativas respecto a sus pares heterosexuales. Estos hallazgos van en la línea de los resultados obtenidos en otros estudios, en los que se veía que la población no heterosexual, tanto mayor como en general, tenían una mayor afectación emocional debido a su orientación sexual (Carroll, 2015; Espada et al., 2012; Fredriksen-Goldsen \& Kim, 2015; Fredriksen-Goldsen et al., 2013; Gonzales \& Henning-Smith, 2015; Marchueta, 2014; Martxueta \& Etxeberria, 2014; Reisner et al., 2014; Seelman, 2018).

En cuanto al tipo de malestar, se observa que la población mayor no-heterosexual tiende a sentirse más rechazada o sola, al igual que en pasadas investigaciones (Fredriksen-Goldsen et al., 


\section{MALESTAR EMOCIONAL ASOCIADO A LA ORIENTACIÓN SEXUAL EN PERSONAS MAYORES}

2013). Si bien en este estudio, las diferencias son menores, podría ser debido a la disimilitud entre los participantes puesto que, en este caso, solamente respondieron aquellas personas heterosexuales y no heterosexuales que habían sufrido malestar a causa de su orientación y no toda la población en general.

Así pues, aunque sea en menor porcentaje, también se observan ciertas dudas y malestar entre la población mayor heterosexual. Este hecho podría relacionarse con su propia vivencia de la heterosexualidad que quizá no correspondería a la visión normativa que impera. Como indican pasados estudios, dentro del propio colectivo heterosexual, hay personas que manifiestan haber tenido fantasías o sueños eróticos homosexuales (Morales-Knight \& Hope, 2012; Yu, 2013) 0, incluso, haber tenido relaciones sexuales con alguien de su mismo sexo (Copen, Chandra \& Febo-Vazquez, 2016; Vrangalova \& Savin-Williams, 2012), lo que podría ser fuente de dudas o malestar.

En cualquier caso, respecto a la violencia sufrida, se observa que la población no-heterosexual ha sido, en mayor medida, víctima de violencia verbal y física debido a su orientación sexual, en comparación con sus pares heterosexuales, lo que iría en consonancia con pasados estudios (D’Augelli \& Grossman, 2001; Herek, 2009). Estos resultados serían consecuencia del rechazo social existente contra la homosexualidad (Kuyper, ledema \& Keuzenkamp, 2013; Smith, Son \& Kim, 2014).

Pese a la importancia de estos hallazgos, también hay que remarcar algunas limitaciones de este estudio. Por una parte, habría que aumentar el número de participantes, para obtener así unos resultados más concluyentes. De ese modo, se podrían hacer análisis entre diversas orientaciones sexuales minoritarias, para conocer si existen patrones diferenciales entre ellas. También sería conveniente realizar un estudio parecido con diferentes intervalos de edad, para ver si existen diferencias en la afectación emocional en función de la misma. Finalmente, los resultados se verían enriquecidos con un estudio de corte cualitativo en el que se pudiera preguntar a esas personas cuál ha sido la vivencia de su orientación sexual a lo largo de su vida y cuáles, los principales factores clave en esa vivencia.

De cualquier manera, mediante estos resultados, se ha comprobado el rechazo social que sufren las personas mayores con una orientación sexual no normativa, así como las diferencias con la población heterosexual en cuanto a las dudas y malestar relacionado con la vivencia de la propia orientación. Por ello, con el fin de mejorar la situación que vive este sector de la población, habría que estudiar qué variables podrían mediar en estos resultados, para realizar programas de prevención e intervención adecuados. Por ejemplo, podría incluirse en los programas estrategias que mejorasen la red de apoyo, ya que se ha observado que ayuda a rebajar los niveles de soledad y mejora la calidad de vida de las personas mayores no-heterosexuales (Fredriksen-Goldsen et al., 2012; Fredriksen-Goldsen, Kim, Shiu, Goldsen \& Emlet, 2014; Kuyper \& Fokkema, 2010).

\section{REFERENCIAS}

Asociación Internacional de Lesbianas, Gays, Bisexuales, Trans e Intersex (2017). Homofobia de Estado 2017. Estudio jurídico mundial sobre la orientación sexual en el derecho: criminalización, protección y reconocimiento. Recuperado el 10 febrero, 2018, de: http://ilga.org/downloads/2017/ILGA_Homofobia_de_Estado_2017_WEB.pdf

Carroll, J. L. (2015). Sexuality now: Embracing diversity (5th edition). EE UU: Cengage Learning.

Copen, C. E., Chandra, A. \& Febo-Vazquez, I. (2016). Sexual behavior, sexual attraction, and sexual orientation among adults aged 18- the United States: Data from the 2011-2013 National Survey of Family Growth. National Health Statistics Reports, (88), 1-14.

D’Augelli, A. R. \& Grossman, A. H. (2001). Disclosure of sexual orientation, victimization, and men- 
tal health among lesbian, gay, and bisexual older adults. Journal of Interpersonal Violence, 16(10), 1008-1027.

DePalma, R. \& Jennett, M. (2010). Homophobia, transphobia and culture: Deconstructing heteronormativity in English primary schools. Intercultural Education, 21(1), 15-26.

Diamond, L. M. \& Huebner, D. M. (2012). Is good sex good for you? Rethinking sexuality and health. Social and Personality Psychology Compass, 6(1), 54-69.

Digoix, M., Franchi, M., Pichardo Galán, J. I., Selmi, G., de Stéfano Barbero, M., Thibeaud, M. \& Vela, J. A. M. (2016). Sexual orientation, family and kinship in France, Iceland, Italy and Spain. Families and Societies Working Paper 54(2016). Recuperado el 13 Mayo, 2017, de: http://www.familiesandsocieties.eu/wp-content/uploads/2016/08/WP54Digoixetal2016.pdf

Enson, S. (2015). Causes and consequences of heteronormativity in healthcare and education. British Journal of School Nursing, 10(2), 73-78.

Espada, J. P., Morales, A., Orgilés, M. \& Ballester, R. (2012). Autoconcepto, ansiedad social y sintomatología depresiva en adolescentes españoles según su orientación sexual. Ansiedad y Estrés, 18(1), 31-41.

Fredriksen-Goldsen, K. I., Emlet, C. A., Kim, H. J., Muraco, A., Erosheva, E. A., Goldsen, J. \& HoyEllis, C. P. (2012). The physical and mental health of lesbian, gay male, and bisexual (LGB) older adults: The role of key health indicators and risk and protective factors. The Gerontologist, 53(4), 664-675.

Fredriksen-Goldsen, K. I. \& Kim, H. J. (2015). Count me in: Response to sexual orientation measures among older adults. Research on Aging, 37(5), 464-480.

Fredriksen-Goldsen, K. I., Kim, H. J., Barkan, S. E., Muraco, A. \& Hoy-Ellis, C. P. (2013). Health disparities among lesbian, gay, and bisexual older adults: Results from a population-based study. American Journal of Public Health, 103(10), 1802-1809.

Fredriksen-Goldsen, K. I., Kim, H. J., Shiu, C., Goldsen, J. \& Emlet, C. A. (2014). Successful aging among LGBT older adults: Physical and mental health-related quality of life by age group. The Gerontologist, 55(1), 154-168.

Gonzales, G. \& Henning-Smith, C. (2015). Disparities in health and disability among older adults in same-sex cohabiting relationships. Journal of Aging and Health, 27(3), 432-453.

Hayman, B., Wilkes, L., Halcomb, E. \& Jackson, D. (2013). Marginalised mothers: Lesbian women negotiating heteronormative healthcare services. Contemporary Nurse, 44(1), 120-127.

Herbenick, D., Reece, M., Schick, V., Sanders, S. A., Dodge, B. \& Fortenberry, J. D. (2010). Sexual behavior in the United States: results from a national probability sample of men and women ages 14-94. The journal of sexual medicine, 7(5), 255-265.

Herek, G. M. (2009). Hate crimes and stigma-related experiences among sexual minority adults in the United States: Prevalence estimates from a national probability sample. Journal of Interpersonal Violence, 24(1), 54-74.

Herek, G. M. \& McLemore, K. A. (2013). Sexual prejudice. Annual Review of Psychology, 64, 309333.

Herz, M. \& Johansson, T. (2015). The normativity of the concept of heteronormativity. Journal of Homosexuality, 62(8), 1009-1020.

Kuyper, L. \& Fokkema, T. (2010). Loneliness among older lesbian, gay, and bisexual adults: The role of minority stress. Archives of Sexual Behavior, 39(5), 1171-1180.

Kuyper, L., ledema, J. \& Keuzenkamp, S. (2013). Towards tolerance. Exploring changes and explaining differences in attitudes towards homosexuality in Europe. The Hague: The Netherlands Institute for Social Research. 


\section{MALESTAR EMOCIONAL ASOCIADO A LA ORIENTACIÓN SEXUAL EN PERSONAS MAYORES}

Lindau, S. T. \& Gavrilova, N. (2010). Sex, health, and years of sexually active life gained due to good health: Evidence from two US population based cross sectional surveys of ageing. BMJ: British Medical Journal, 340, c810.

Lindau, S. T., Schumm, L. P., Laumann, E. O., Levinson, W., O'muircheartaigh, C. A. \& Waite, L. J. (2007). A study of sexuality and health among older adults in the United States. New England Journal of Medicine, 357(8), 762-774.

McNeill, T. (2013). Sex education and the promotion of heteronormativity. Sexualities, 16(7), 826846.

Marchueta, A. (2014). Consecuencias del bullying homofóbico retrospectivo y los factores psicosociales en el bienestar psicológico de sujetos LGB. Revista de Investigación Educativa, 32(1), 255-271.

Martxueta, A. \& Etxeberria, J. (2014). Análisis diferencial retrospectivo de las variables de salud mental en lesbianas, gais y bisexuales (LGB) víctimas de bullying homofóbico en la escuela. Revista de Psicopatología y Psicología Clínica, 19(1), 23-35.

Morales-Knight, L. F. \& Hope, D. A. (2012). Correlates of same-sex attractions and behaviors among self-identified heterosexual university students. Archives of Sexual Behavior, 41(5), 1199-1208.

Morgan, E. M., Thorne, A. \& Zurbriggen, E. L. (2010). A longitudinal study of conversations with parents about sex and dating during college. Developmental Psychology, 46(1), 139-150.

Morrison, S. \& Dinkel, S. (2012). Heterosexism and health care: A concept analysis. Nursing Forum, 47(2), 123-130.

Reisner, S. L., Biello, K., Perry, N. S., Gamarel, K. E. \& Mimiaga, M. J. (2014). A compensatory model of risk and resilience applied to adolescent sexual orientation disparities in non-suicidal self-injury and suicide attempts. American Journal of Orthopsychiatry, 84(5), 545-546.

Röndahl, G. (2011). Heteronormativity in health care education programs. Nurse Education Today, 31(4), 345-349.

Seelman, K. L. (2018). Differences in Mental, Cognitive, and Functional Health by Sexual Orientation Among Older Women: Analysis of the 2015 Behavioral Risk Factor Surveillance System. The Gerontologist, $O(0), 1-11$.

Smith, T. W., Son, J. \& Kim, J. (2014). Public attitudes toward homosexuality and gay rights across time and countries (pp. 1-29). Los Angeles, CA: The Williams Institute.

Vrangalova, Z. \& Savin-Williams, R. C. (2012). Mostly heterosexual and mostly gay/lesbian: Evidence for new sexual orientation identities. Archives of Sexual Behavior, 41(1), 85-101.

Wynn, L. L., Foster, A. M. \& Trussell, J. (2009). Can I get pregnant from oral sex? Sexual health misconceptions in e-mails to a reproductive health website. Contraception, 79(2), 91-97.

Yu, C. K. C. (2013). Lust, pornography, and erotic dreams. Dreaming, 23(3), 175-193. 